\title{
Acute extracellular fluid volume changes increase ileocolonic resistance to saline flow in anesthetized dogs
}

A.T. Santiago Jr. F. de-A.A. Gondim, D.I.M. Cavalcante, J.R.V. da-Graça, G.R. de-Oliveira, A.A. dos-Santos and F.H. Rola
Departamento de Fisiologia e Farmacologia,

Universidade Federal do Ceará, 60430-270 Fortaleza, CE, Brasil

\section{Correspondence \\ F.H. Rola \\ Departamento de Fisiologia \\ e Farmacologia, UFC \\ Rua Coronel Nunes de Melo, 1127 \\ Caixa Postal 3157 \\ 60430-270 Fortaleza, CE \\ Brasil \\ Fax: 55 (085) 243-9333}

Presented at the IX Annual Meeting of the Federação de Sociedades de Biologia Experimental, Caxambu, MG, Brasil, August 24-27, 1994.

This work is part of a Master's thesis presented by A.T. Santiago Jr. to the Departamento de Farmacologia, Universidade Federal do Ceará, Fortaleza, CE.

Research supported by CNPq, CAPES and UFC.

Received March 21, 1996 Accepted June 6, 1997

\section{Abstract}

We determined the effect of acute extracellular fluid volume changes on saline flow through 4 gut segments (ileocolonic, ileal, ileocolonic sphincter and proximal colon), perfused at constant pressure in anesthetized dogs. Two different experimental protocols were used: hypervolemia ( $i v$ saline infusion, $0.9 \% \mathrm{NaCl}, 20 \mathrm{ml} / \mathrm{min}$, volume up to $5 \%$ body weight) and controlled hemorrhage (up to a 50\% drop in mean arterial pressure). Mean ileocolonic flow $(\mathrm{N}=6)$ was gradually and significantly decreased during the expansion $(17.1 \%, \mathrm{P}<0.05)$ and expanded $(44.9 \%, \mathrm{P}<0.05)$ periods while mean ileal flow $(\mathrm{N}=7)$ was significantly decreased only during the expanded period $(38 \%, \mathrm{P}<0.05)$. Mean colonic flow $(\mathrm{N}=7)$ was decreased during expansion $(12 \%$, $\mathrm{P}<0.05)$ but returned to control levels during the expanded period. Mean ileocolonic sphincter flow $(\mathrm{N}=6)$ was not significantly modified. Mean ileocolonic flow $(\mathrm{N}=10)$ was also decreased after hemorrhage (retracted period) by $17 \%(\mathrm{P}<0.05)$, but saline flow was not modified in the other separate circuits $(\mathrm{N}=6,5$ and 4 for ileal, ileocolonic sphincter and colonic groups, respectively). The expansion effect was blocked by atropine $(0.5 \mathrm{mg} / \mathrm{kg}$, iv) both on the ileocolonic $(\mathrm{N}=6)$ and ileal $(\mathrm{N}=5)$ circuits. Acute extracellular fluid volume retraction and expansion increased the lower gastrointestinal resistances to saline flow. These effects, which could physiologically decrease the liquid volume being supplied to the colon, are possible mechanisms activated to acutely balance liquid volume deficit and excess.

\section{Introduction}

The luminal fluid in the gastrointestinal (GI) tract is in dynamic equilibrium with the extracellular fluid (ECF) volume (1). Acute ECF volume retraction by hemorrhage, dehydration or reduction in salt intake increases intestinal salt and water absorption (2). In
Key words

- Ileocolonic motility

- Extracellular fluid volume

- Expansion

- Hemorrhage

- Atropine 
expansion by intravenous infusion of saline also decreases both gastric and jejunal compliance while hemorrhage increases it $(6,7)$. These observations suggest that the GI tract, at least in its upper portions, behaves like a regulatory liquid reservoir capable of adjusting its volumetric, absorptive and secretory capacities to cope with organic needs, i.e., accommodating and absorbing more liquid volume after hemorrhage and, contrarily, becoming less receptive to liquid volume distension and less absorptive after acute hypervolemia. This evidence is supported by the functional interaction between motor changes and absorption; increased tone reduces absorption while decreased tone stimulates salt and water absorption (8).

However, despite the contributions of the ileal and large intestine to liquid volume regulation (9), the effect of ECF volume changes on the lower GI tract motor behavior has not been studied. Under basal conditions, i.e., when the large bowel absorbs about $20 \%$ of the total ingested fluids and gut secretion (10), patients with ileostomies are more susceptible to dehydration when placed on a low sodium diet or during an acute diarrheal illness (11). Therefore, we decided to study the effect of acute ECF volume expansion and retraction on the resistance to liquid flow offered by the ileocolonic segment as a whole, as well as by its segments in anesthetized dogs.

\section{Material and Methods}

\section{Animals and surgical procedures}

Mongrel dogs of either sex $(12.7 \pm 3.2$ $\mathrm{kg}, \mathrm{N}=62$ ) fasted for $16 \mathrm{~h}$ with free acess to water were utilized. The animals were anesthetized with sodium pentobarbital $(30 \mathrm{mg} /$ $\mathrm{kg}, i v)$ and the femoral vessels were cannulated. The right vein was used for anesthetic infusion when necessary or for isotonic saline $(0.9 \% \mathrm{NaCl})$ infusion to expand the ECF volume. The right artery was utilized for bleeding and to provide blood samples for arterial hematocrit determinations. The left artery was cannulated with a heparinized ( 35 $\mathrm{IU} / \mathrm{ml}$ ) glass cannula and connected to an $\mathrm{Hg}$ manometer. Mean arterial pressure (MAP) and breathing were recorded with a Palmer kymograph. Central venous pressure (CVP) in $\mathrm{cmH}_{2} \mathrm{O}$ was measured visually using a catheter introduced via the external jugular vein, positioned in the right atrium and connected to an $\mathrm{H}_{2} \mathrm{O}$ manometer zeroed with the normal ictus cordis.

\section{Perfusion circuits}

After laparotomy, one of the following 4 different types of perfusion circuits was created (Figure 1).

A - Ileocolonic segment perfusion. Figure $1 \mathrm{~A}(\mathrm{~N}=16,13 \pm 3 \mathrm{~kg})$.

An oral probe $(0.8 \mathrm{~cm}$ ID, $1.0 \mathrm{~cm}$ OD) introduced through an ileal fistula was positioned and fixed with an obstructive ligature in the distal ileum. An aboral probe $(1.0 \mathrm{~cm}$ ID, $1.3 \mathrm{~cm} \mathrm{OD}$ ) introduced through a colonic fistula was positioned and fixed with another obstructive ligature in the proximal colon. The tips of both cannulas were positioned $4 \mathrm{~cm}$ from the ileocolonic sphincter. Care was taken to preserve regional circulation and innervation in all perfusion groups.

$B$ - Ileal perfusion. Figure 1B $(\mathrm{N}=13$, $13.4 \pm 2.2 \mathrm{~kg}$ ).

The oral probe $(0.8 \mathrm{~cm} \mathrm{ID,} 1.0 \mathrm{~cm} \mathrm{OD)}$ was introduced through an ileal fistula (15 $\mathrm{cm}$ from the ileocolonic sphincter), and the tip was positioned approximately $10 \mathrm{~cm}$ from the ileocolonic sphincter. The aboral probe (1.0 cm ID, $1.3 \mathrm{~cm} \mathrm{OD)} \mathrm{introduced} \mathrm{through}$ a colonic fistula $(2 \mathrm{~cm}$ from the ileocolonic sphincter) was positioned in the terminal ileum, near the ileocolonic sphincter.

C-Ileocolonic sphincter perfusion. Figure $1 \mathrm{C}(\mathrm{N}=11,12.8 \pm 2.9 \mathrm{~kg})$.

The oral probe $(0.8 \mathrm{~cm} \mathrm{ID,} 1.0 \mathrm{~cm} \mathrm{OD)}$ was introduced through an ileal fistula $(5 \mathrm{~cm}$ from the ileocolonic sphincter), and the tip 


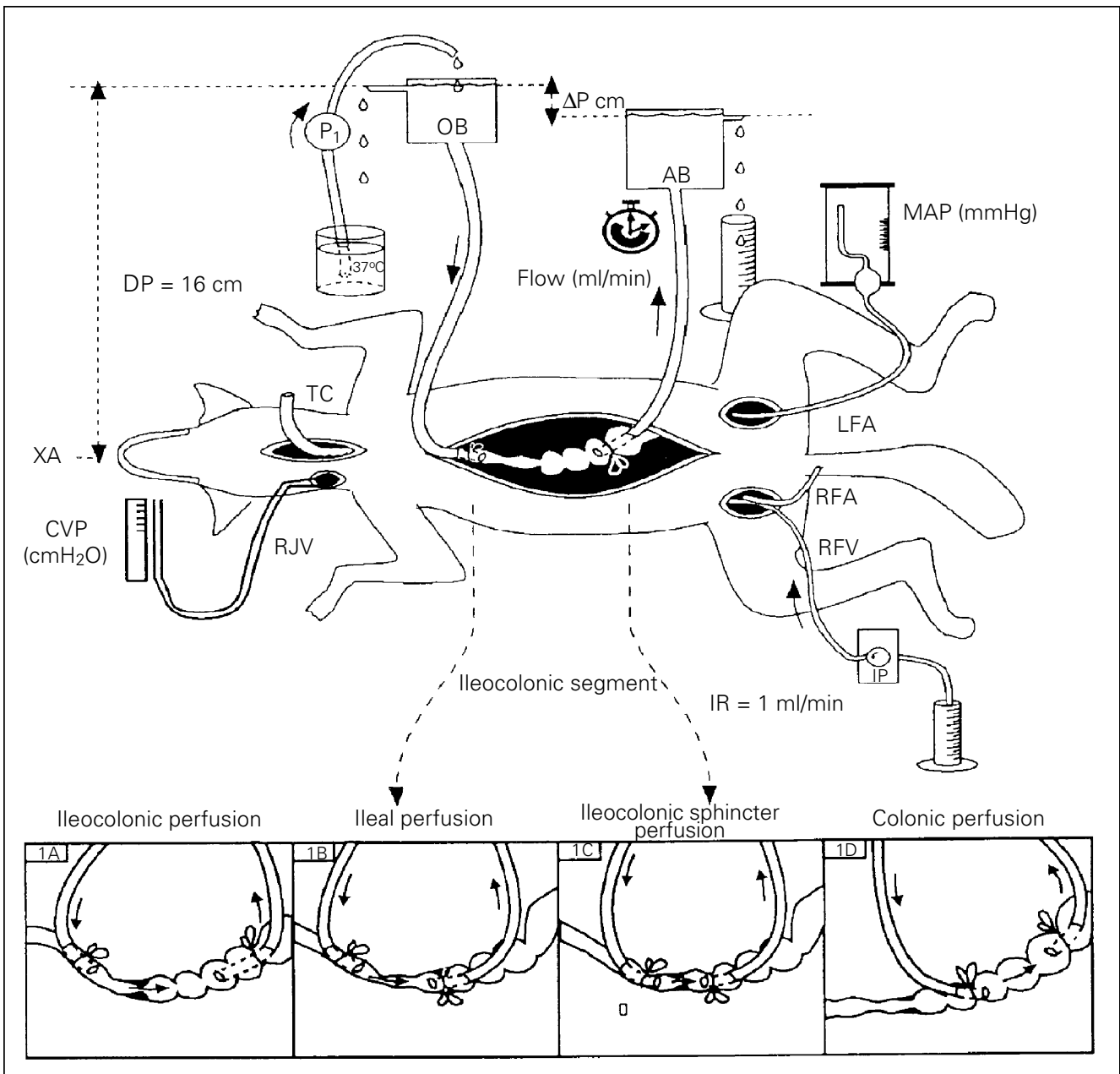

was positioned and fixed near the ileocolonic sphincter. The aboral probe $(1.0 \mathrm{~cm}$ ID, $1.3 \mathrm{~cm} \mathrm{OD}$ ) introduced through a colonic fistula ( $5 \mathrm{~cm}$ from the ileocolonic sphincter) was positioned in the terminal ileum, near the ileocolonic sphincter.

$D$ - Colonic perfusion. Figure 1D $(\mathrm{N}=11$, $14 \pm 3.6 \mathrm{~kg}$ ).

The oral probe $(0.8 \mathrm{~cm} \mathrm{ID,} 1.0 \mathrm{~cm} \mathrm{OD)}$ was introduced through a colonic fistula near the ileocolonic sphincter and the tip was positioned and fixed $2 \mathrm{~cm}$ from the ileocolonic sphincter. The aboral probe $(1.0 \mathrm{~cm}$ ID, $1.3 \mathrm{~cm}$ OD) introduced through a colonic fistula $(10 \mathrm{~cm}$ from the ileocolonic sphincter) was positioned in the proximal colon, 8 $\mathrm{cm}$ from the ileocolonic sphincter.

The free end of each probe (oral and aboral) was connected to the barostat bottom which was then filled with isotonic saline solution $(0.9 \% \mathrm{NaCl}, \mathrm{w} / \mathrm{v})$ and maintained at a constant liquid level throughout the experiment (Figure 1). The oral barostat liquid level, placed $16 \mathrm{~cm}$ above the xiphoid appendix, was always kept above the aboral barostat in order to ensure an aboral ileocolonic flow due to the resulting pressure gradient. The result was a communicating vessel system in which the perfused segment represented the only resistance offered to the liquid flow which could vary. The liquid flowing out of the aboral barostat was manually collected at 2-min intervals and measured and the rate is reported as the ileocolonic, ileal, ileocolonic sphincter and colonic flows in $\mathrm{ml} / \mathrm{min}$. To obtain an ileoco-
Figure 1 - Schematic representation of the barostat system utilized to perfuse the ileocolonic (1A), ileal (1B), ileocolonic sphincter (1C) and colonic (1D) portions of the gastrointestinal tract under constant pressure in anesthetized dogs. The oral barostat (OB) liquid level was positioned $16 \mathrm{~cm}$ (DP, distension pressure) above the xiphoid appendix (XA). A pressure gradient $(\Delta \mathrm{P})$ to promote an aboral saline flow was obtained by positioning the aboral barostat ( $A B$ ) below the OB. The volume of saline at $37^{\circ} \mathrm{C}$ flowing out of the $A B$ was manually collected at 2min intervals and is reported as the mean flow value obtained during control and experimental protocols. $\mathrm{P}_{1}$ and IP, Peristaltic pumps used to replenish OB liquid level and for saline infusion and blood reinfusion, respectively; RJV, right jugular vein for central venous pressure measurements (CVP, $\mathrm{CmH}_{2} \mathrm{O}$ ); RFA, right femoral artery for arterial pressure measurements (MAP, $\mathrm{mmHg}$ ); LFA, left femoral artery for bleeding; RFV, right femoral vein for anesthetic or saline infusion; IR, infusion rate; TC, tracheal cannula. 
lonic flow of about $50 \mathrm{ml} / \mathrm{min}$ and $70 \mathrm{ml} / \mathrm{min}$ in the other separate segments (ileal, ileocolonic sphincter and colonic segments) a variation in the pressure gradient was performed: $4.7 \pm 0.3,3.4 \pm 1.3,2.5 \pm 0.6$ and $1.6 \pm 0.5$ $\mathrm{cmH}_{2} \mathrm{O}$, respectively. The isotonic saline utilized in the perfusing system was heated to $37^{\circ} \mathrm{C}$ before reaching the gut by circulating it in a glass coil immersed in a water bath at $38^{\circ} \mathrm{C}$.

\section{Experimental design}

After surgery, the animals were allowed to rest for a period of 20-30 min to stabilize hemodynamic and respiratory parameters. After this interval, the mean rate of saline flowing from the aboral barostat over a period of 20 min represented the normovolemic control flow. The animals were submitted to acute ECF volume expansion by $i v$ isotonic saline infusion $(0.9 \% \mathrm{NaCl}$ solution, $20 \mathrm{ml} / \mathrm{min}$ ) up to a volume equivalent to $5 \%$ of the body weight or acute ECF volume retraction by controlled hemorrhage until a $50 \%$ drop in MAP levels, followed by reinfusion of the removed blood at a rate of $20 \mathrm{ml} / \mathrm{min}$. The perfusion flow was measured during saline infusion (expansion period), $30 \mathrm{~min}$ after it was completed (expanded period), during hemorrhage and 20 min thereafter (ECF volume retraction and retracted, respectively), as well as during blood volume reinfusion (ECF volume restoration).

At the end of each experiment, the animals were sacrificed under deep anesthesia by $i v \mathrm{KCl}$ injection.

\section{Drugs}

In another set of experiments, atropine sulfate (Sigma Chemical Co., St. Louis, MO) was injected $i v(0.5 \mathrm{mg} / \mathrm{kg})$ after normovolemic control into ileal $(\mathrm{N}=5)$ and ileocolonic $(\mathrm{N}=6)$ perfusion circuits. Saline flow measurements were then recorded for $30 \mathrm{~min}$ (drug control) and ECF volume expansion was performed according to the previous protocol.

\section{Statistical analysis}

The results are reported as mean \pm SEM. One-way repeated measures ANOVA and Dunnett's test were used to compare the differences in saline flow between each experimental period and the Student $t$-test for paired means was used to compare percent differences in hematocrit. Statistical differences were considered to be significant at $\mathrm{P}<0.05$.

\section{Results}

Effect of acute ECF volume expansion on ileocolonic, ileal, colonic and ileocolonic sphincter segment perfusion flow, MAP, CVP and hematocrit

Figure 2 shows the effect of acute expansion on the ileocolonic (2A), ileal (2B), ileocolonic sphincter (2C) and colonic (2D) segment flow, MAP and CVP. As can be seen in Figure 2A, the mean ileocolonic flow $(\mathrm{N}=6)$ was gradually decreased during the expansion period until the difference was statistically significant when a volume up to $5 \%$ body weight was infused $(\mathrm{P}<0.05)$, persisting during the first $20 \mathrm{~min}$ of the expanded period $(\mathrm{P}<0.05)$. The mean percent flow reductions during the expansion and expanded periods were 23 and $45 \%$, respectively (from $46.7 \pm 2.8$ to $38.7 \pm 4.4$ and $25.7 \pm 6.3 \mathrm{ml} /$ min, respectively, $\mathrm{P}<0.05)$. MAP was not significantly modified while CVP levels increased at the beginning of expansion (1\% body weight), remaining stable at least during the first $20 \mathrm{~min}$ of the expanded period $(\mathrm{P}<0.05)$.

Figure 2B shows that the mean ileal flow $(\mathrm{N}=7)$ slowly decreased during the expansion period, reaching a statistically significant difference only during the expanded 
A
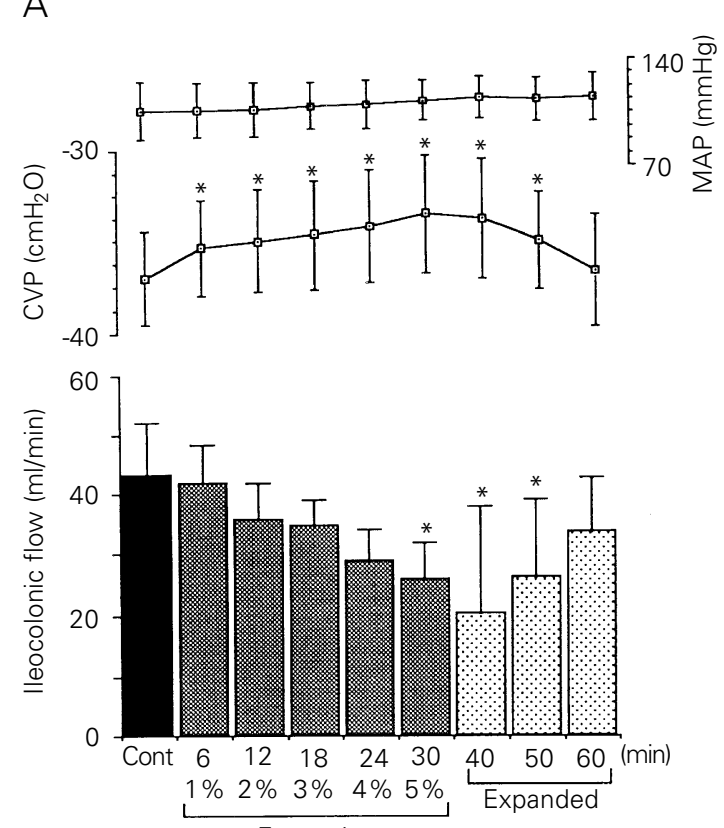

C

Expansion

C (\% body weight)
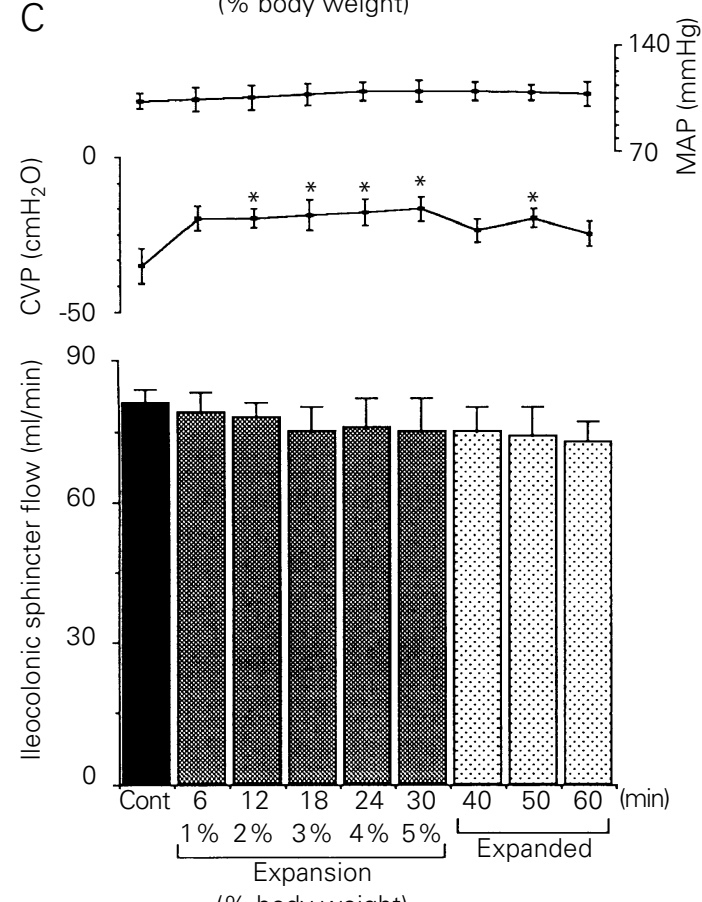

(\% body weight)
B
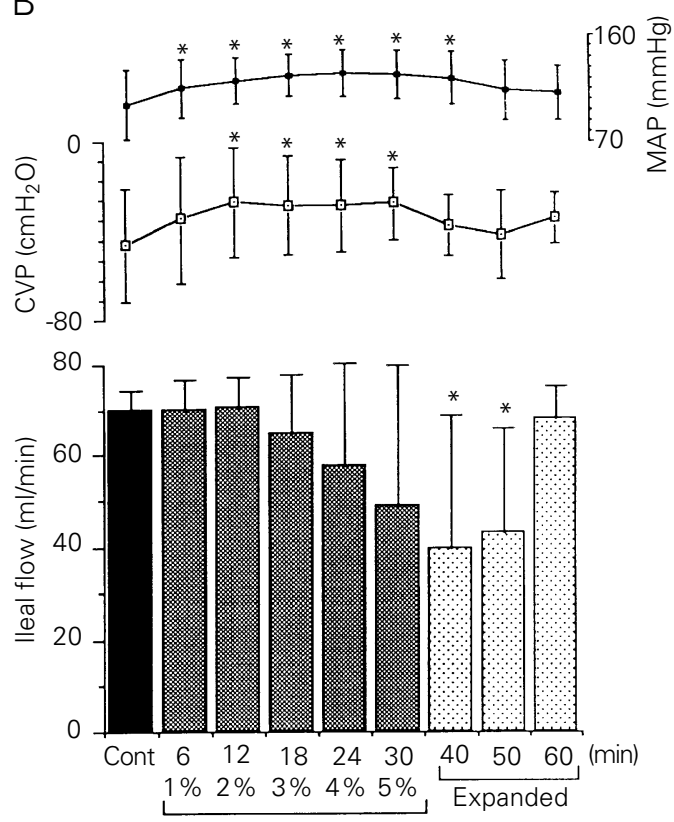

Expansion

(\% body weight)
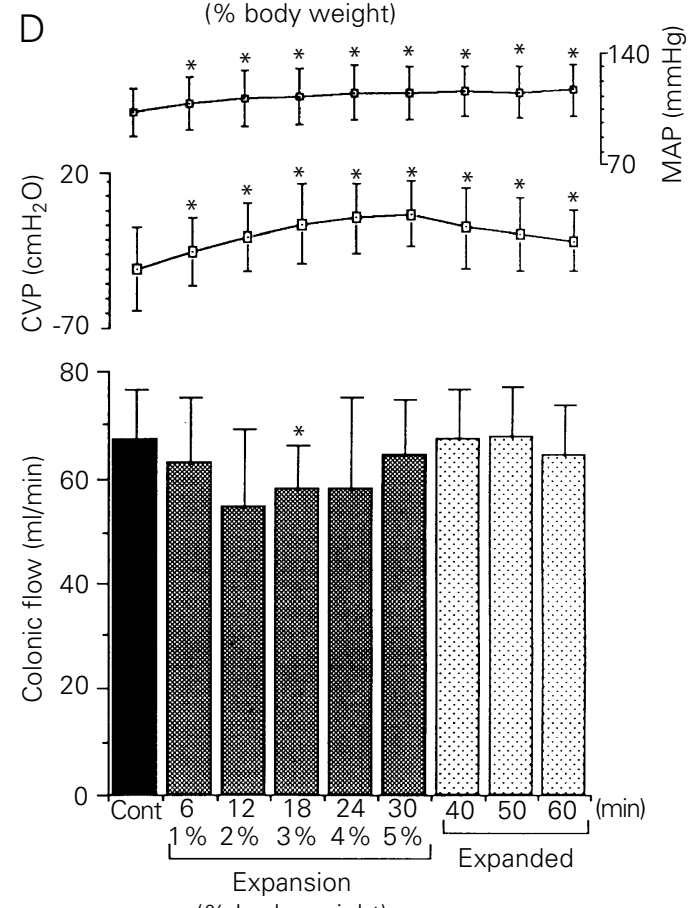

(\% body weight)

Figure 2 - Effect of acute extracellular fluid (ECF) volume expansion on the ileocolonic (A), ileal (B), ileocolonic sphincter (C) and colonic (D) flows of saline (in $\mathrm{ml} / \mathrm{min}$ ), central venous pressure (CVP) and mean arterial pressure (MAP) of anesthetized dogs. After a normovolemic control period (20 min), the animals were expanded by iv isotonic saline infusion $(20 \mathrm{ml} / \mathrm{min}$ ) up to $5 \%$ body weight. The saline flow was monitored during expansion and 30 min thereafter. Bars represent the mean values of saline flow during each period. The expansion period was increased stepwise at $1 \%$ intervals from 1 to $5 \%$ body weight and is reported for each step. The expanded period was divided into three 10-min intervals. Cont, Normovolemic control period. Upper dots indicate MAP (mmHg) and lower dots $\mathrm{CVP}\left(\mathrm{cmH}{ }_{2} \mathrm{O}\right)$. Vertical lines indicate standard error of the mean. ${ }^{*} \mathrm{P}<0.05$ compared to control period (Dunnett's test). 
period $(\mathrm{P}<0.05)$, and remaining stable for at least $20 \mathrm{~min}$. The mean percent flow reductions in the expansion and expanded periods were 19.3 and $38 \%$, respectively (from $70 \pm$ 4.4 to $56.5 \pm 8.5$ and $43.4 \pm 11.7 \mathrm{ml} / \mathrm{min}$, respectively, $\mathrm{P}<0.05$ ). CVP levels increased significantly during expansion when a volume up to $2 \%$ body weight was infused, remaining increased until the end of expansion $(\mathrm{P}<0.05)$. MAP levels increased significantly throughout the expansion period and during the first $10 \mathrm{~min}$ of the expanded pe$\operatorname{riod}(\mathrm{P}<0.05)$.

The mean ileocolonic sphincter flow $(\mathrm{N}$ $=6$ ) was not significantly modified either during the expansion or during the expanded period (from $81 \pm 2.7$ to $76.4 \pm 6.4$ and 73.8 $\pm 5.2 \mathrm{ml} / \mathrm{min}$, Figure 2C). MAP was also not significantly modified. Even when we analyzed the changes in flow separately on the basis of the percent body weight of volume infused (as in the ileocolonic and ileal flows), no significant flow reduction was observed. However, CVP levels were significantly increased throughout the expansion and expanded periods $(\mathrm{P}<0.05)$.

Figure 2D shows that the mean colonic flow $(\mathrm{N}=7)$ decreased during the expansion and expanded periods, but attained statistical significance only during expansion and not during the expanded period. The mean percent flow decrease during the expansion

Table 1 - Effect of ECF volume expansion and retraction on arterial hematocrit.

Expansion was obtained by isotonic saline infusion of $1 \%$ to $5 \%$ stepwise at $20 \mathrm{ml} /$ min. Retraction was obtained by bleeding until there was a $50 \%$ reduction in arterial pressure. Data are reported as means \pm SEM for 51 dogs. Number of animals in each group reported in parenthesis. ${ }^{*} \mathrm{P}<0.01$ compared to control values (Student $t$-test).

\begin{tabular}{lccc}
\hline Perfusion group & Control & Expanded & Retracted \\
\hline Ileocolonic & $43.3 \pm 3.3$ & $29.3 \pm 3.0^{*}$ & $40.8 \pm 4.5$ \\
& $(16)$ & $(6)$ & $(10)$ \\
Ileal & $43.9 \pm 5.6$ & $33.8 \pm 8.2^{*}$ & $44.2 \pm 3.8$ \\
& $(13)$ & $(7)$ & $(6)$ \\
Ileocolonic sphincter & $43.9 \pm 6.8$ & $33.3 \pm 8.5^{*}$ & $38.0 \pm 10.1$ \\
& $(11)$ & $(6)$ & $(5)$ \\
Colonic & $40.6 \pm 8.6$ & $35.1 \pm 7.0^{*}$ & $35.5 \pm 9.0$ \\
& $(11)$ & $(7)$ & $(4)$
\end{tabular}

period was $12 \%$ (from $67.7 \pm 3.4$ to $59.8 \pm$ 3.5 and $66.3 \pm 3.9 \mathrm{ml} / \mathrm{min}, \mathrm{P}<0.05)$. MAP and CVP were significantly increased throughout the expansion and expanded periods $(\mathrm{P}<0.05)$.

Table 1 shows that the arterial hematocrit values were significantly decreased during the expanded period when compared to control values $(\mathrm{P}<0.001)$.

\section{Effect of atropine infusion on acute ECF volume expansion, ileocolonic and ileal flows, MAP and CVP}

Figure 3 shows that atropine injection blocked the effect of ECF volume expansion on the ileocolonic (3A) and ileal saline flows (3B). Atropine per se did not modify saline flow during the atropine control period (from $68.9 \pm 5.2$ to $71.4 \pm 4.9$ and from $64.2 \pm 3.3$ to $64.6 \pm 2.7 \mathrm{ml} / \mathrm{min}$ ) and no additional saline flow decrease was observed during the expansion or expanded periods (to $64.6 \pm$ 7.5 and $70.5 \pm 4.8 \mathrm{ml} / \mathrm{min}$ and to $64.3 \pm 3.2$ and $61.8 \pm 2.9 \mathrm{ml} / \mathrm{min}$, for ileocolonic and ileal perfusions, respectively). MAP and CVP levels were significantly increased during the expansion and expanded periods $(\mathrm{P}<0.05)$ and MAP levels were also increased during the atropine control period in the ileal perfusion group $(\mathrm{P}<0.05)$.

After expansion, in the atropinized animals, the arterial hematocrit decreased from $45.6 \pm 6.0$ and $35.7 \pm 6.6$ to $29.8 \pm 3.6$ and $25.5 \pm 2.6 \%$ in the ileocolonic sphincter and ileal perfusion flows, respectively $(\mathrm{P}<0.001)$.

\section{Effect of acute ECF volume retraction on ileocolonic, ileal, colonic and ileocolonic sphincter segment flows, MAP and CVP}

Figure 4 shows the effect of acute ECF volume retraction on the ileocolonic (4A), ileal (4B), ileocolonic sphincter (4C) and colonic (4D) segment flow, MAP and CVP.

Ileocolonic flow was significantly decreased by $17 \%$ after ECF volume retraction 


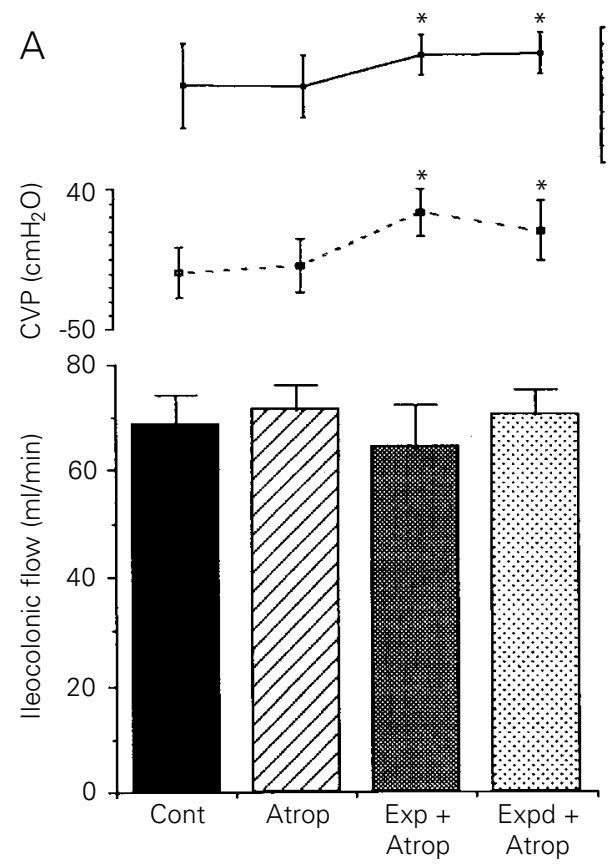

during the retracted period (from $52.8 \pm 3.9$ to $45.5 \pm 5 \mathrm{ml} / \mathrm{min}, \mathrm{P}<0.05$ ), but returned to control levels during removed blood reinfusion (Figure 4A). Figure 4B, 4C and 4D show that saline flow was not modified during or after retraction (retracted period) or after blood volume reinfusion in the other perfusion groups. MAP levels decreased during and after retraction and during removed blood volume reinfusion $(\mathrm{P}<0.05)$ in the ileocolonic, ileocolonic sphincter and colonic perfusion groups. In ileal perfusion, however, MAP returned to control levels after reinfusion of removed blood. CVP levels were decreased during and after retraction in the ileocolonic and colonic groups $(\mathrm{P}<0.05)$ and only during retraction in the ileocolonic sphincter group $(\mathrm{P}<0.05)$ but were not modified in the ileal group.

Table 1 shows that acute ECF volume retraction did not modify the arterial hematocrit values.

\section{Discussion}

We have previously demonstrated that acute volume expansion increases gas-
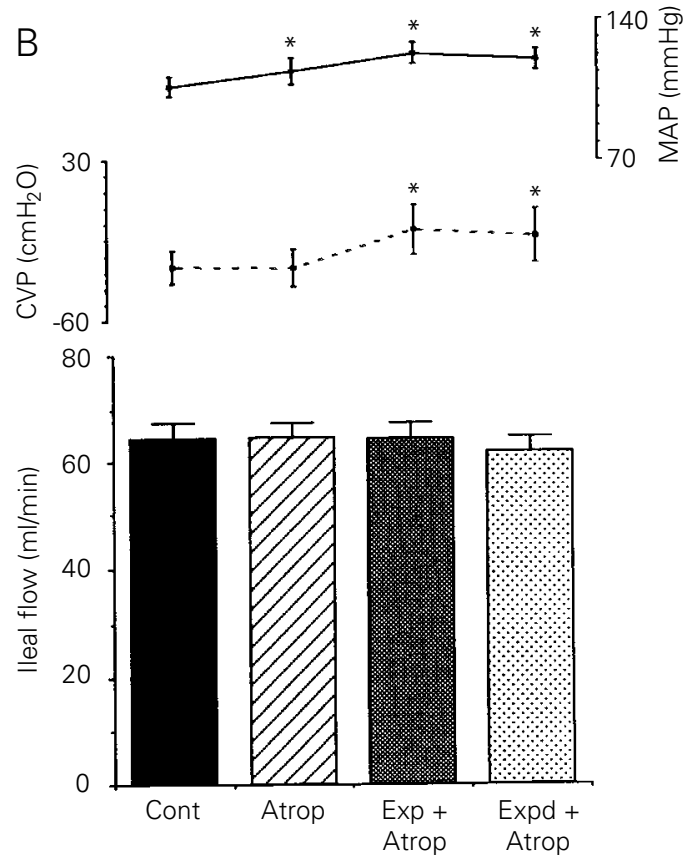

troduodenal resistance to saline flow in rats and dogs, while hypovolemia due to hemorrhage decreases it $(4,5)$. Since ECF volume retraction increases intestinal salt and water absorption (2) while expansion reduces or even abolishes intestinal absorption and increases secretion (3), we proposed a role for ECF volume in the modulation of liquid flow through the upper GI tract, i.e., coupled modulation of the GI tract contractile activity, salt and water transport and ultimately luminal liquid transit to cope with organic needs.

The present study, using the same experimental protocol (ileocolonic segment and separate ileal, ileocolonic sphincter and colonic portions were perfused under constant pressure and changes in flow were assumed to represent modifications in motor activity, tone and/or motility), extends this idea to the lower gut, showing that acute volume imbalances also modify the motor behavior of ileocolonic segments. This approach has been frequently utilized to study resistances through discrete portions of the gut $(4,5,12)$.

In constrast to the opposite flow changes (reduction and increase) which we observed after opposite volume imbalances
Figure 3 - Effect of atropine on acute extracellular fluid (ECF) volume expansion, ileocolonic (A) and ileal (B) saline flow $(\mathrm{ml} / \mathrm{min})$, central venous pressure (CVP) and mean arterial pressure (MAP) of anesthetized dogs. After normovolemic control period (Cont, $20 \mathrm{~min}$ ), the animals received an $i v$ injection of atropine $(0.5$ $\mathrm{mg} / \mathrm{kg}$ ). The perfusion saline flow was measured for 30 min (Atrop) after the animals were expanded by $i v$ isotonic saline infusion $(20 \mathrm{ml} / \mathrm{min})$ up to $5 \%$ body weight. The perfusion flow was monitored during expansion (Exp) and for 30 min thereafter (Expd). Bars represent the mean perfusion flow values. Continuous lines represent MAP $(\mathrm{mmHg})$ and broken lines represent CVP $\left(\mathrm{cmH}_{2} \mathrm{O}\right)$. Vertical lines indicate standard error of the mean. ${ }^{*} P<0.05$ compared to control period (Dunnett's test). 
Figure 4 - Effect of acute extracellular fluid (ECF) volume retraction on the ileocolonic (A), ileal (B), ileocolonic sphincter (C) and colonic (D) flows of saline, central venous pressure (CVP) and mean arterial pressure (MAP) of anesthetized dogs. After a normovolemic control period $(20$ $\mathrm{min})$, the ECF volume was retracted by bleeding the animals up to a $50 \%$ drop in MAP. The animals were then brought back to the "euvolemic" condition by reinfusing the blood volume previously removed at a rate of 20 $\mathrm{ml} / \mathrm{min}$. Besides the normovolemic control period, ileocolonic, ileal, ileocolonic sphincter and colonic saline flows $(\mathrm{ml} / \mathrm{min})$ were measured during bleeding and for a 20-min period thereafter (ECF volume retraction and retracted, respectively) as well as during autologous blood reinfusion (restoration). Bars represent average of flow values during the normovolemic control (Cont), retraction (Ret), ECF volume retracted (Retd) and restoration (Rest) periods. Continuous and interrupted lines represent MAP $(\mathrm{mmHg})$ and $\mathrm{CVP}\left(\mathrm{cmH}_{2} \mathrm{O}\right)$, respectively. Vertical lines indicate the standard error of the mean. ${ }^{*} P<0.05$ compared to control period (Dunnett's test).

(expansion and retraction) in the gastroduodenal segment (4), both acute ECF volume expansion and retraction were associated with decreased ileocolonic saline flow.

Similar to the gastroduodenal segment, ECF volume expansion gradually and persistently decreased the saline flow through the ileocolonic segment during the expan-

A
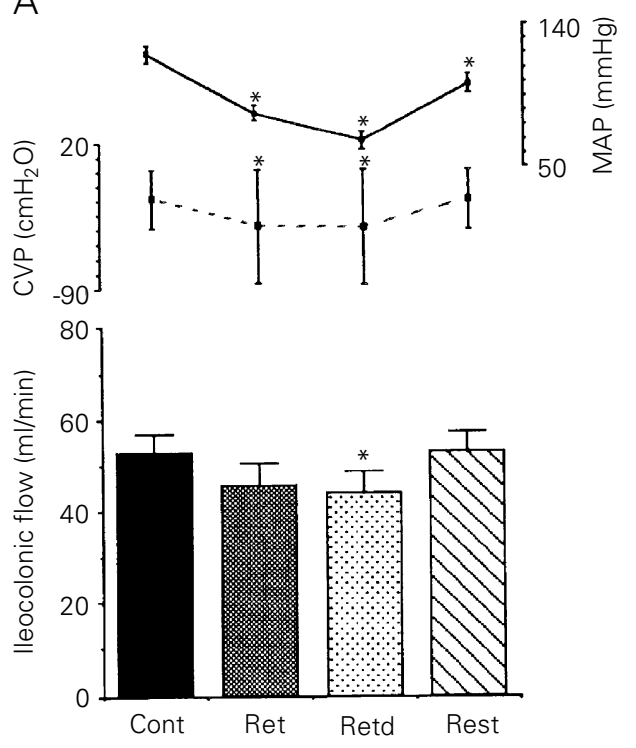

C
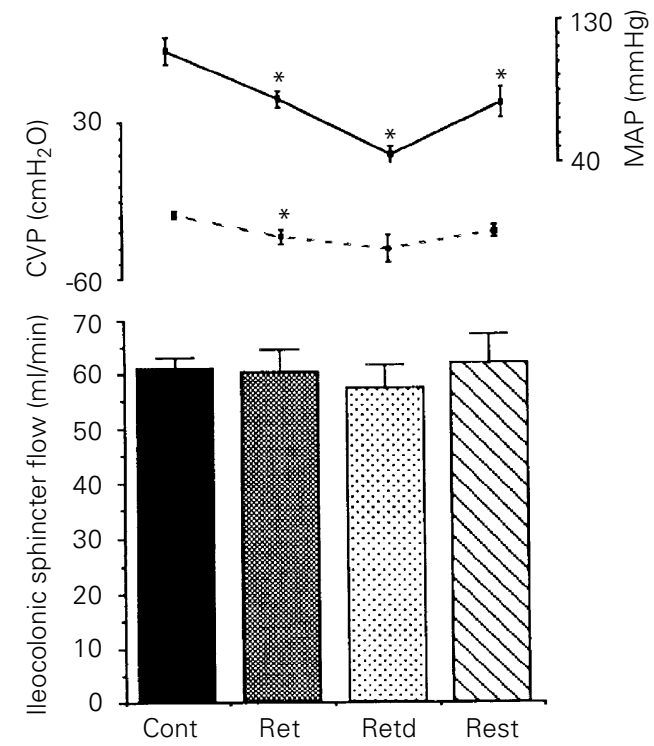

sion and expanded periods. However, when searching for the main sites of resistance, we observed some peculiarities. In the separate ileal perfusion, we observed a late saline flow decrease, which was significant only during the expanded period. In contrast, saline flow in the separate ileocolonic sphincter was quite stable both during the expansion and expanded peri-

B
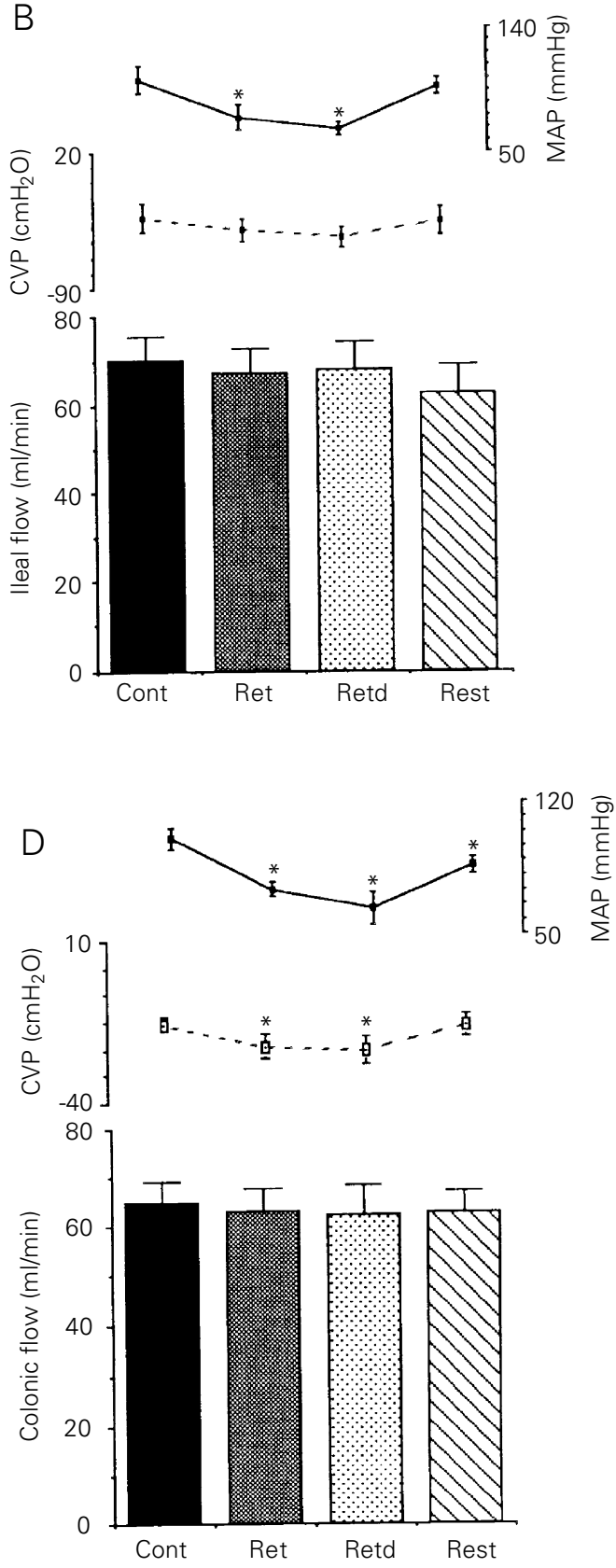
ods, while colonic flow reduction was slight and more pronounced only during the expansion period. Thus, analyzing Figure 2, the flow decrease observed in Figure $2 \mathrm{~A}$ appears to be the final result of the sum contribution of Figure 2B and 2D. These findings point to the ileum as the major contributor to the marked flow reduction observed during the expanded period while colonic segment contribution appears to be especially important for the earlier flow decrease during the expansion period, observed throughout ileocolonic segment perfusion.

In contrast to the increased gastroduodenal flow after ECF retraction that we have previously observed (5), ECF volume retraction, like expansion, was followed by a smaller but still significant drop in ileocolonic flow. However, when we attempted to identify the main sites of resistance we were unable to find them since no saline flow decrease either during or after ECF volume retraction was observed in the other separate perfusion circuits (ileal, ileocolonic sphincter and colonic circuits). These findings suggest that the integrated sum of the ileal, ileocolonic sphincter and colonic resistances appears to be necessary for the complete expression of the ECF volume retraction effect on the ileocolonic segment.

In addition to reducing saline flow, ECF volume expansion induced hemodilution and increased CVP and MAP (except in ileocolonic perfusion). ECF volume retraction was also followed by major circulatory changes, with MAP and CVP being significantly reduced. Hematocrit values were decreased after ECF volume expansion but remained unchanged immediately after hemorrhage. These findings suggest that modifications in CVP levels are closely related to saline flow changes, but MAP was not modified in the ileocolonic perfusion group, where a significant saline flow reduction was observed. In addition, an opposite effect was observed, i.e., ileocolonic flow was reduced while CVP and MAP were increased simultaneously.

ECF volume expansion with isotonic, isotonic-isooncotic or isotonic-isooncoticisohemic infusion produced similar reductions in gastroduodenal flow (4). These results indicate that the effect of hypervolemia on gut motility does not appear to be related to factors other than acute volemic expansion.

The cholinergic role in the decrease in saline ileocolonic and ileal flows due to acute ECF volume expansion was further investigated. For this purpose, the effect of atropine (which is a competitive muscarinic antagonist in smooth muscle) was studied. Atropine per se did not modify ileal or ileocolonic flows. In addition, no saline flow change was observed during or after ECF volume expansion in animals pretreated with atropine. Thus, the effect of acute ECF volume expansion was blocked by atropine. Since atropine decreases intestinal contractile activity (13), the saline flow decrease due to acute ECF volume expansion in the lower GI tract may involve an intestinal contractile activity increase by cholinergic activation, which was antagonized by atropine.

In summary, in contrast to our previous results in the upper GI tract portions (4-7), we observed that both opposite volemic changes (expansion and retraction) increased the ileocolonic resistance to saline flow. The physiological relevance of these findings is speculative and yet to be established, especially because of the small number of supporting reports in this field. However, the decrease of liquid volume being supplied to the colon could indicate that the small bowel is more apt to promptly deal with acute volemic challenges. This idea is reinforced by the results of other studies, which demonstrate increased small bowel fluid and electrolyte absorption during retraction (2), decreased 
absorption/increased secretion during expansion (3) and apparent large bowel incapacity to readily and efficiently modify those patterns under the same volemic conditions (14). Consequently, the persistence of basal ileocolonic transit could contribute to further aggravate liquid volume excess or deficit.

\section{Acknowledgments}

We are grateful to the Zoonoses Center of the City of Fortaleza for providing the experimental animals (street dogs) and to Dr. R.T. Fogaça for statistical advice.

\section{References}

1. Miller WL \& Dale HE (1978). Restoration of haemorrhaged plasma volume by gastrointestinal fluid in dogs. American Journal of Physiology, 234: H80-H87.

2. Levens NR (1985). Control of intestinal absorption by the renin-angiotensin system. American Journal of Physiology, 249: G3-G15.

3. Duffy PA, Granger DN \& Taylor AE (1978). Intestinal secretion induced by volume expansion in dogs. Gastroenterology, 75: 413-418.

4. Xavier-Neto J, Dos Santos AA \& Rola FH (1990). Acute hypervolemia increases the gastroduodenal resistance to the flow of saline in rats. Gut, 31: 1006-1010.

5. Santos AA, Xavier-Neto J, Santiago Jr AT, Souza MAN, Martins AS, Alzamora F \& Rola FH (1991). Acute volaemic changes modify the gastroduodenal resistance to the flow of saline in anaesthetized dogs. Acta Physiologica Scandinavica, 143: 261269.
6. Capelo LR, Cavalcante DM, Leitão IA, Cristino-Filho G \& da Silva EAT (1983). Modifications of gastric compliance in dogs related to changes of extracellular fluid volume. Brazilian Journal of Medical and Biological Research, 16: 73-76.

7. Rola FH, Santos AA, Xavier-Neto J, Cristino-Filho G, Rocha Cl, Santiago Jr AT, Gondim FAA, Pereira JM \& Capelo LR (1989). Effects of acute volemic changes on jejunal compliance in dogs. Brazilian Journal of Medical and Biological Research, 22: 523-531

8. Lee JS (1983). Relationship between intestinal motility, tone, water absorption and lymph flow in the rat. American Journal of Physiology, 345: 489-499.

9. Clarke AM, Miller M \& Shields R (1987). Intestinal transport of sodium, potassium, and water in dogs during sodium depletion. Gastroenterology, 83: 846-858.
10. Binder HJ, Sandle GI \& Rajendran VM (1991). Colonic fluid and electrolyte transport in health and disease. In: Phillips SF, Pemberton JH \& Shorter RG (Editors), The Large Intestine. Chapter 7. Raven Press, New York, 141-168.

11. Wright WK, Cleveland JC \& Tilson MD (1969). Morphology and absorptive capacity of the ileum after ileostomies in man. American Journal of Surgery, 117: 242245.

12. Jarret RJ \& Gazet JC (1966). Studies in vivo of the ileocaecocolic sphincter in the cat and the dog. Gut, 7: 217-225.

13. Jahnberg $T$, Abrahamsson $H$, Haglund $M$, Jansson G \& Martisson J (1976). Vagotomy and gastric reservoir function. European Surgical Research, 2 (Suppl): 6970.

14. Higgins Jr JT \& Blair NP (1971). Intestinal transport of water and electrolytes during extracellular volume expansion in dogs. Journal of Clinical Investigation, 50: 25692579. 